\title{
Service Delivery Location Postal Address
}

National Cancer Institute

\section{Source}

National Cancer Institute. Service Delivery Location Postal Address. NCI Thesaurus. Code C95395.

The postal address of the service delivery location. 